\title{
La dimensión humana de la información: biblioteca e inclusión social en la América Latina
}

\author{
The human dimension of information: libraries and social inclusion in South America
}

\author{
Prof. Dr. Emir José Suaiden \\ Departamento de Ciência da Informação, Universidade de Brasília, Brasil
}

\begin{abstract}
Resumen
Se evidencia la dimensión social del conocimiento, traducida en la integración de su dimensión humana y tecnológica, con el objetivo de contribuir al desarrollo social de toda la nación. Cuando una sociedad carece de un espíritu crítico que le permita rebelarse contra la falta de políticas y estructuras informacionales, sólo resta, para cambiar la situación existente, el apoyo decisivo de los profesionales de la información. El trabajo con comunidades marginadas exige mucho esfuerzo, comprensión, liderazgo y el uso de metodologías adecuadas. El empresario, el académico, el ciudadano incluido en la sociedad de la información, deben encarar ese tipo de trabajo como un desafío, y, ante todo, como un rescate de su deuda social. La falta de lectores, de ciudadanos críticos, se debe, según los sociólogos, al hecho de que hayamos pasado rápidamente de una cultura oral a una cultura audiovisual, fenómeno que enfatiza la necesidad del mediador en el proceso de inclusión. En el Brasil, y no obstante los enormes avances económicos ocurridos, se dejaron de realizar reformas y de tomar decisiones políticas que impidieran el enfrentamiento de los problemas asociados a la concentración de riqueza y a la exclusión social. Como consecuencia, la manipulación de conceptos tan simplistas como el de estar o no alimentado, ser o no alfabetizado, tener o no una ocupación con rendimiento adecuado, comenzó a mostrarse insuficiente para abordar el proceso de exclusión. También se tornó inadecuada la simple enumeración cuantitativa del acceso a la educación, al trabajo, a la información, etc., debiendo acoplar la noción de calidad del acceso a los bienes y servicios públicos. El concepto de exclusión social está siendo adoptado, de manera creciente, para dar cuenta de un fenómeno que se manifiesta en forma cada vez más compleja. En el panorama que se anuncia, no puede aceptarse ya la exclusión ni la simple aceptación de la miseria. Integrar a los excluidos es la semántica de la solidaridad. No obstante, las actividades antisolidarias aún encuentran eco y condiciones favorables para su proliferación. Reman contra esa corriente las apuestas a favor del actual desarrollo tecnológico y de las redes interactivas que lo acompañan, multiplicándose en redes de solidaridad. La dimensión social del conocimiento juega un papel central en ese proceso. Representa la alianza
\end{abstract}

entre la academia, el gobierno, la iniciativa privada y la sociedad civil organizada. El papel de la academia es fomentar la investigación, crear bases teóricas, desarrollar metodologías adecuadas y conocimientos que contribuyan a la solución de los problemas nacionales. El papel del Gobierno es formular políticas públicas, crear una infraestructura para favorecer la inclusión social y la difusión del conocimiento. El papel de la iniciativa privada es el rescate de su responsabilidad social. El papel del Tercer Sector es facilitar las acciones de todos los actores antes citados. Es ésta la única alianza que puede generar un esfuerzo común capaz de implantar una política de justicia social, de crear una conciencia solidaria abocada a minimizar las desigualdades sociales, y de ampliar en el Brasil la sociedad de la información.

Palabras clave: Bibliotecas Dimensión social. Inclusión social. América Latina.

\begin{abstract}
In the fields os education, culture and social references Latin America is characterized as a region of great social exclusion. A large portion of the population is excluded from the information society, a factor that creates much violence and instability due to the lack of job opportunities. The present work proposes a new model of information dissemination to include people in the information society. The base to this is empowering libraries and forming a reading public. Another proposition is the use of methods such as: information mediation, information literacy, informational competences and above all the interaction among the bibliographic, the virtual and the digital for the construction of quality education.
\end{abstract}

Keywords: Libraries. Social aspects. Social inclusion. South America. 


\section{Introducción}

En cada período histórico las sociedades humanas tienen elementos que caracterizan la base de su organización social. Cuando esos elementos se convierten en principios de acción, o en principios organizadores del comportamiento humano o del modo en que funcionan las organizaciones y las instituciones sociales, se constituyen en valores que caracterizan su entorno social y el período histórico que están construyendo. Creado por la necesidad humana de entender y explicar el medio en que se vive, es éste el papel que la información y el conocimiento desempeñan hoy en el mundo.

Las sociedades que, gracias a procesos continuos de generación y uso del conocimiento, logran organizarse de tal modo que puedan contribuir al proceso del aprendizaje social, son clasificadas como sociedades del conocimiento. Tienen ellas capacidad de generar conocimiento sobre su realidad y su entorno, y condiciones de utilizarlo en su proceso de concebir y construir su futuro. De este modo, el conocimiento se transforma, no sólo en instrumento para comprender y explicar la realidad, sino también en motor de desarrollo y factor dinámico de los cambios sociales.

EI XXI es el siglo de la tecnología y de la información, época en la que el conocimiento es el elemento central de la estructura económica que está surgiendo, y la innovación el principal vehículo para transformar en valor el conocimiento. La creación de éste y su incorporación a innovaciones tecnológicas son instrumentos cruciales para el desarrollo sostenible de cualquier nación. Es fácil constatar que el movimiento transformador, que hoy apunta a la información, la comunicación y la propia educación, constituye una profunda revolución tecnológica. Basta para ello recordar el hecho de que un estudio de la Unión Europea — La sociedad de la información, Bruselas, 1996 - afirma que en los últimos veinte años se han duplicado los conocimientos científicos, que corresponden hoy a la totalidad de saberes técnicos acumulados durante toda la historia de la humanidad.

Vivimos en un mundo interconectado, donde las proporciones de los sistemas globalizados de comunicación superaron los pronósticos más optimistas. Cuando Arthur Clarke, valga el ejemplo, concibió en 1945 la idea de satélites estacionarios, pensaba que no sería posible desarrollarlos antes del año 2000. No obstante, bastaron dos décadas para poner en órbita el Early Bird, luego denominado Intelsat I. Así que cuando Neil Armstrong descendió en la luna, en otro escenario de ficción, millones de personas pudieron verlo pisar un terreno que antes sólo era posible recorrer en las fantasías de escritores y artistas de hace muchos siglos.

Fue ése el inicio de las comunicaciones globalizadas, que están transformando las civilización contemporánea. La muy elogiada Internet, red mundial de computadores, es el centro de una revolución que está afectando profundamente nuestros modos de comunicación, de aprendizaje, de trabajo y de gobierno. Por otro lado están avanzando las aplicaciones que hacen realidad las reflexiones de visionarios como Buckminster Fuller y J. C. R. Licklider, quienes, hace algunas décadas, teorizaron sobre la posibilidad de desarrollar sistemas automáticos de interacción con el conocimiento.

Castells (1994) hace referencia a un proceso de transformación estructural de las sociedades avanzadas, vinculado a la revolución tecnológica, a la globalización económica y cultural y a la habilidad de crear conocimiento y de procesar información. La idea es que la productividad y el crecimiento económico, así como la generación de riquezas y de poder, se estructuran socialmente sobre el control del conocimiento y de la información.

\section{Información y Conocimiento en la América Latina del siglo XXI}

El conocimiento y la información pasan a cobrar nueva importancia en ese contexto. La revolución tecnológica, característica primera del nuevo milenio, transformó la realidad, modificó los valores que caracterizaron las últimas décadas del siglo $\mathrm{XX}$, extinguió profesiones, creó otras, $\mathrm{y}$ tornó imperativa una nueva forma de organización. Dejó claro que necesitamos replantear nuestros conceptos y, por encima de todo, conceptuar de una manera nueva antiguos valores.

Cuando pensamos hoy en información y conocimiento, es preciso mirar esos valores bajo la óptica de la educación, de la economía y de la cultura. Es fundamental identificar la dimensión humana, donde la educación es la base del asunto; la dimensión tecnológica, donde el factor económico reviste esencial importancia; y la dimensión social, donde la cultura se presenta como resultado de las transformaciones experimentadas por la sociedad.

El proceso de transformación es global, abarca a todos los países directa o indirectamente, con mayor o menor intensidad. En los países desarrollados, la sociedad del conocimiento surge como consecuencia natural del proceso de desarrollo. En América Latina, en especial en el Mercosur, así como en todos los países en de- 
sarrollo, se hace necesaria la adopción de estrategias políticas, educacionales y sociales, traducidas en programas y acciones que posibiliten la inclusión de estas sociedades en el mundo globalizado.

La implantación de la sociedad de la información en el Mercosur es un tema actual, y cada vez más debatido en las estructuras gubernamentales y en los eventos científicos. La pregunta más discutida es ésta: ¿es posible establecer a medio plazo en la región la sociedad de la información? Para responder a ese interrogante es de suma importancia analizar las estructuras educacionales, económicas y sociales del Mercosur, y al mismo tiempo compararlas con las exigencias de la propia sociedad de la información.

La sociedad de la información trae en su núcleo los mismos indicadores de las sociedades anteriores, tales como poder adquisitivo, nivel educacional y lenguaje. La diferencia radica en su necesidad de acceso a la información, sea bibliográfica o virtual. Cuando se analizan las estructuras informacionales puede comprobarse que son deficientes porque, con excepción de Brasil y Argentina, no existe una industria editorial fuerte ni, en consecuencia, un público lector adecuado, lo cual torna deficiente la formación de contenidos para la sociedad de la información. Cuando un país en vía de desarrollo importa muchos contenidos, por carecer de ellos, es esto un indicador de que es dependiente y seguirá siendo dominado. Hasta que esa realidad no sea transformada, no tendrá una producción científica y tecnológica apta para responder a un proceso de desarrollo.

\section{Educación y desarrollo en América Latina}

La estructura educacional y social en general hoy existente en la América Latina y en el Mercosur es aún muy precaria, y contribuye al alto índice de analfabetismo, desnutrición infantil y desempleo de la región. En muchas localidades el gran aliciente que lleva al alumno a frecuentar la escuela es la merienda escolar, no el sistema educacional. Son pocas las escuelas con bibliotecas, y algunas de ellas están a cargo de profesores que nada saben al respecto. Cuando hay biblioteca, el acervo se compone por lo general de libros didácticos y de referencia, siendo escasos los títulos representativos de literatura infantil y juvenil.

La investigación es siempre el aspecto más abandonado del sistema educacional, pues se basa a efectos prácticos en la copia de diccionarios y enciclopedias. Si se cuenta con un compu- tador, la investigación consiste en copiar textos de la Internet. Los contenidos programáticos de los cursos se basan muchas veces en apostillas y libros desactualizados. Generalmente los profesores están mal remunerados, y su bajo nivel de autoestima acaba por ser transferido a los alumnos. El panorama general es una mezcla de falta de preparación, desinformación y dependencia. No suele haber coordinación pedagógica, hay falta de planeación y de dialogo entre los profesores, lo cual hace inviable el tema de la interdisciplinaridad. La formación de los maestros es precaria, y no existen por lo general programas adecuados de capacitación. En los últimos años la violencia ha crecido de manera asustadora en las escuelas, y hay ya casos de alumnos armados en el recinto escolar, y de agresión a profesores. Los alumnos de mayor poder adquisitivo abandonan la escuela pública para ingresar e establecimientos privados.

Las causantes del fracaso escolar son la falta de capacitación del profesor — pues en algunas regiones los maestros no ostentan título alguno-, la carencia de infraestruturas de laboratorio, de biblioteca escolar y de contenidos didácticos adecuados a la región. Todos esos problemas acaban afectando el aprendizaje del alumno, quien muchas veces no logra interpretar un texto mínimo ni organizarse intelectualmente para entender, valga el ejemplo, contenidos de Matemática o Física. Esta cultura de la desinformación, de la manipulación y de la dependencia hace posible la concentración de capitales, la desigualdad social y la exclusión.

En un rápido análisis sobre la dimensión económica del Mercosur fue posible verificar que, especialmente en los últimos años, ha aumentado la desigualdad, pues la riqueza se halla concentrada en una minoría. Estudios recientes comprueban que una parte significativa de la población que pertenecía a la clase media es hoy parte de la clase pobre, pues hay un declive cada vez mayor de las clases sociales. El alto índice de desempleo estimula el mercado informal, donde muchas veces la mercancía dominante es la piratería, con total falta de respeto a la creación intelectual y a la ética que rige los derechos autorales. Realidad fundada en la necesidad de supervivencia, y para muchos la única forma de acceso a los bienes culturales.

La verdad es que la información aún no es accesible para gran parte de la población de los países en vía de desarrollo. Muchas generaciones se levantaron ignorando qué significa el acceso a la escuela, a las bibliotecas escolares, a las públicas, a las juveniles e infantiles, al teatro, al cine y al ocio. Juzgaban esas instituciones elitistas y propias de una cultura erudita. 
Sin embargo, la biblioteca puede transformar la calidad de vida de las comunidades, repartiendo información adecuada con la rapidez que exigen los tiempos modernos. Dicho trabajo debe partir de un diagnóstico bien elaborado sobre las necesidades de información de la comunidad, para luego, con el auxilio de técnicas conducentes a la toma de decisiones, elaborar una planeación estratégica compatible con la realidad local.

Como no logramos desarrollar una cultura bibliográfica, aún hoy la información que circula en esos países es la información oral, obtenida informalmente en la iglesia, en el trabajo, en los medios de comunicación y en la escuela. Tanta informalidad lleva al individuo a acostumbrarse también a la educación informal, y luego al trabajo informal, asuntos por completo incompatibles con la filosofía de la sociedad del conocimiento. En la medida en que no existe en la región una política informativa que dé privilegio a la inclusión social, este papel deberá ser realizado por el profesional de la información, que habrá de trabajar como mediador para hacer posible el compartir del conocimiento.

\section{Dimensión social del conocimiento}

Cuando hablamos de dimensión social del conocimiento estamos identificando un nivel de conciencia colectiva que exige una mejor distribución de los saberes y de las riquezas generados por la sociedad. Es un llamado a la necesidad de disminuir las desigualdades y socializar el bienestar, la calidad de vida, la ciudadanía y la dignidad humana. En ese sentido, es necesaria una gran movilización de la sociedad, donde cada órgano, institución, empresa e individuo trabajen por la equidad social. De ese modo es posible concebir la construcción de una nueva mentalidad, que redundará en la construcción de una cultura representativa de una sociedad más justa, basada en la unión solidaria de la tecnología, la información y el conocimiento.

Profundizando un poco más en el debate sobre la dimensión social del conocimiento, vemos que la cultura formada a partir de la revolución tecnológica exige una mayor responsabilidad social al gobierno, a las empresas y a los individuos partícipes de la sociedad globalizada. Para dejar más claro el concepto de la dimensión social del conocimiento, trataremos de analizarlo bajo el punto de vista práctico: cómo aparece en la educación, en el mercado y en el gobierno.

En la educación, por medio de proyectos de inclusión digital trabajados en las escuelas, bibliotecas o en cualquier acción educacional. En este caso, es básico que el educador tenga clara su función de mediador, para que sus en- señanzas puedan ir más allá de los contenidos programáticos ofrecidos por los gobiernos competentes. Es indispensable mostrar a los educandos su papel dentro del contexto en que viven, en tanto que actores sociales del momento histórico que su generación está construyendo. La dimensión social del conocimiento en el ámbito de la educación, sea ésta formal o informal, es producto de la capacidad crítica, competencia, compromiso y colaboración de los educadores y los educandos.

La biblioteca debería ser el gran instrumento de la educación, especialmente en lo que atañe a la dimensión social del conocimiento. Tradicionalmente, las bibliotecas han sido creadas por decretos, $y$, sin relación alguna con la realidad de la comunidad donde funcionan, no son visibles para ésta. Además de invisibles, no causan ningún impacto social en las organizaciones que, teóricamente, deberían actuar como difusoras y multiplicadoras de una política educacional, cultural y social. La mejor prueba de esto es la constante falta de presupuesto en las actividades de difusión de la información.

En el ámbito del mercado es posible advertir un movimiento tendiente a hacer visible la dimensión social del conocimiento. Las empresas se están manifestando acerca de ese asunto, por medio de la responsabilidad social que cada vez está más presente en el marketing institucional $y$ en las inversiones en proyectos que contemplan una mejoría en la calidad de vida de las comunidades menos favorecidas. Son muchos los proyectos de inclusión social y digital patrocinados, valga el ejemplo, por la iniciativa privada. Otro indicador que ratifica ese dato es una mayor aproximación entre la academia y las empresas, en el sentido de desarrollar investigaciones que agreguen valor a sus productos, para atender mejor a las demandas de la sociedad. Esta nueva postura del mercado nace de la dimensión social del conocimiento, y es una señal de que estamos, en el caso brasilero, marchando en dirección a la sociedad de la información.

En lo que respecta a la sociedad, esa dimensión social del conocimiento se materializa por medio de las Organizaciones No Gubernamentales (ONGs), las Organizaciones de la Sociedad Civil de Interés Público (OSCIPs), y de las asociaciones y cooperativas, que han crecido considerablemente en los últimos años. Dichas instituciones están enfocadas sobre la dimensión social del conocimiento en la medida en que sus trabajos se desarrollan, de modo general, a partir de un interés en compartir conocimientos, experiencias y saberes en busca de una mejoría de la calidad de vida en la comunidad donde actú- 
an. Es posible observar que el concepto de colaboración y su efectivo ejercicio se vieron incrementados y popularizados a partir de esas organizaciones. El concepto que guía las empresas sin fines lucrativos es el desarrollo sostenible, la cualificación profesional, empleo e ingresos, educación, ecología, etc., todos ítems encaminados a la mejora de la calidad de vida, en busca de la equidad social.

En la esfera gubernamental, la dimensión social del conocimiento se visualiza por medio de acciones que conllevan la inclusión social, del apoyo a proyectos que contribuyan a mejorar la cualificación del ciudadano, disminuyendo las desigualdades sociales, y del e-gov que hace disponibles vía web los servicios públicos, sean estos federales, estatales o municipales, de manera práctica, barata y democrática. Estas acciones dejan en claro que la dimensión social del conocimiento, es decir, su apropiación por la sociedad y su reflejo en la formación de la cultura social contemporánea, está presente en la planeación y acciones gubernamentales.

\section{Información, conocimiento y exclusión}

En la complejidad de la sociedad en que vivimos o intentamos vivir, la de la información y el conocimiento, surgen nuevos valores que pasan a caracterizarla. La innovación es un valor fundamental de esa nueva sociedad, y la creatividad es el motor básico en el intento de romper las barreras que protegen el orden establecido, imponiendo cambios, sean ellos sociales, tecnológicos o culturales.

La revolución tecnológica señala un nuevo marco en la historia del desarrollo. Ayuda a romper una serie de paradigmas y trae en su esencia los problemas fundamentales del cambio y la innovación.

El problema del cambio se relaciona íntimamente con los nuevos valores de la sociedad del conocimiento, y aquéllos que no están preparados para enfrentarlos pasan automáticamente a engrosar el porcentaje de excluidos. Sólo es posible cambiar asumiendo una conciencia críti$\mathrm{ca}$, propia de las personas habituadas a la lectura y al uso de la información, lo cual requiere un buen nivel educacional.

Las organizaciones tienen que cambiar para tornarse más competitivas, y sólo lo logran agregando un valor llamado innovación. La innovación crea nuevas fuentes de mercado y fortalece la construcción de la sociedad del conocimiento. Cambio e innovación conducen a la generación de empleo e ingresos, pero contribuyen a am- pliar el mapa de la exclusión, pues en la medida en que surgen nuevas profesiones desaparecen otras, y la exigencia de una mayor calificación es una constante en todos los sectores.

El ciudadano excluido de la sociedad de la información es alguien con baja autoestima, de mirar cabizbajo, poco creativo y en extremo dependiente. No tiene noción de ciudadanía, y generalmente es explotado por la hegemonía reinante. Su comunidad está dominada por la desinformación, es decir, la información está manipulada, y él carece de capacidad crítica para tomar decisiones.

Para combatir ese proceso de desinformación y manipulación de la información, la revolución tecnológica puede ser una gran aliada. Lo que sucede en los países en vía de desarrollo es que un gran porcentaje de la población no tiene acceso a la tecnología, y nunca ha visto un computador. Para esas personas, el acceso y la comprensión deben lograrse mediante metodologías adecuadas de mediación de la información, que comienzan por la alfabetización de la información. En este proceso de inclusión, la dimensión social del conocimiento es el foco que nunca puede perderse de vista.

Los índices de violencia e inseguridad en países pobres son cada vez mayores, y reflejan la desigualdad en la región. Las instituciones que, teóricamente, deberían facilitar el acceso a la sociedad de la información, como la biblioteca y el sistema educacional, no logran, por una serie de factores, alcanzar ese objetivo. Dentro de esa estructura, el papel del profesional de la información cobra extraordinaria importancia, siempre y cuando logren corregirse algunos vicios existentes en la propia formación educacional. Hasta hoy, la mayoría de las escuelas de Biblioteconomía y Ciencia de la Información existentes en la región no privilegian ni la mediación de la información ni la formación de profesionales que sean propagadores de una política de lectura y uso de la información.

\section{La dimensión humana de la información: una línea de investigación}

Para romper las barreras arriba mencionadas, se creó una línea de investigación denominada "Dimensión humana de la información". Este hecho es importante por ratificar las posturas anteriores acerca de la dimensión social del conocimiento. Representa bien los cambios que han de suceder para poder construir, con mayor competencia y rapidez, el puente de acceso al conocimiento que permita atravesar el foso existente entre los países desarrollados y aquellos que están excluidos de la Sociedad de la Información. 
Durante más de una década, y contando con el apoyo del Consejo Nacional de Desarrollo Científico y Tecnológico (CNPq), se realizaron investigaciones sobre el comportamiento de poblaciones marginadas del acceso al libro, la biblioteca y la información.

En los años 90 se iniciaron investigaciones con metodologías apropiadas para comprobar, ante todo, que en las poblaciones marginadas el acceso a la información se ve afectado por la falta de comprensión acerca de lo que ésta significa. A modo de ilustración, citemos el ejemplo de las gentes que habitan en las cercanías de la Plaza de Sé, en Sao Paulo. La información está a su alcance en la Biblioteca Pública Mario de Andrade, muy próxima a la Plaza. La posibilidad de acceso es real, pero la falta de comprensión sobre su significado la torna invisible.

Gran parte de las investigaciones fue realizada en escuelas públicas, y pudo constatarse que biblioteca e información no representan prioridades para el sistema educacional. Los directores de las unidades de enseñanza tuvieron dificultades para percibir que el fracaso escolar -representado por la deserción, la repetición académica y la formación educacional inadecuada - es el mayor indicador de la falta de una eficaz difusión de la información.

El uso indiscriminado del libro didáctico formó una generación que no sabe interpretar el texto leído. Se observó que la investigación escolar quedó restringida a la copia de diccionarios y enciclopedias; que la literatura infantil y juvenil no es estimulada como debería, pues bien se sabe que el interés del estudiante por ese tipo de lectura debe ser despertado por el profesor y por el bibliotecario. Programas como "La hora del cuento", por ejemplo, donde el especialista en lectura aporta la motivación necesaria para la iniciación a la literatura, son fundamentales para la formación de un lector crítico. En las evaluaciones de los proyectos fue posible constatar que ese tipo de acceso a la biblioteca resulta de esencial importancia para la formación de un público lector y es, en consecuencia, un camino viable de inclusión en la Sociedad de la Información. Los proyectos mencionados fueron desarrollados en poblaciones satélites de Brasilia, en regiones de escasos recursos del Distrito Federal.

A partir de los resultados obtenidos en dichas investigaciones, y bajo la perspectiva del uso de recursos tecnológicos, computadores y softwares adecuados, el trabajo fue ampliado y se agregó un nuevo elemento a la inclusión social por medio de la lectura, la inclusión digital. Se añadió a la metodología etnográfica la técnica de la mediación, para construir un modelo de inclusión capaz de responder a las necesidades del ciudadano excluido, y adecuado a la realidad brasilera. Así, se buscó consolidar un modelo de inclusión social, a partir de una acción de inclusión tecnológica, basado en la mediación de la información, mediación contemplada aquí en su relación más estrecha con la difusión, transferencia y transformación de la información en conocimiento.

Compone el modelo la creación de un sistema de indicadores que permita medir los impactos sociales causados por las nuevas tecnologías, tanto en el proceso enseñanza aprendizaje como en la formación del ciudadano y de la comunidad; así como el acompañamiento y la evaluación de sus resultados en un período mínimo de 12 meses. Otro punto importante fue detectar en la actuación del profesional de la información que atiende a la escuela -especialmente el bibliotecario y el monitor que atiende a la comunidad-, en qué momentos y en qué medida la mediación de la información se hace presente y es determinante de aquella actividad. Dicho modelo se concretó en la Escuela Digital Integrada (EDI), y en el concepto de los Centros de Integración Social y Tecnológica (Cistec).

\section{Conclusión}

A lo largo de la historia de la humanidad, el hombre ha venido incorporando nuevos inventos para mejorar su calidad de vida. Por desgracia, estos avances no han llegado de igual modo a todas las personas. Las desigualdades e injusticias sociales han sido y son una constante en la historia del género humano.

El concepto de Tercer Mundo surgió para referirse al mundo subdesarrollado, en contraposición a las sociedades más ricas, que formarían el Primer Mundo. El Segundo Mundo estaría integrado por los países del bloque de economía socialista estatal. Hoy comienza ya a surgir la idea del Cuarto Mundo, compuesto por las manchas negras de la exclusión existente en todo el planeta, presente en cada país y en cada ciudad, en esta nueva geografía de la exclusión social.

Las investigaciones mencionadas consolidaron la comprensión de que, en un modelo de desarrollo marcado por las desigualdades, queda claro que la revolución tecnológica podrá agravar todavía más la exclusión social, si no es adecuadamente conducida. No cabe duda de que enviar computadores a las poblaciones marginadas, sin una propuesta efectiva de mediación de la información, en poco o nada contribuirá a la inclusión de ciudadanos en la sociedad de la información. 
Esta breve retrospectiva evidencia la dimensión social del conocimiento, traducida en la integración de su dimensión humana y tecnológica, con el objetivo de contribuir al desarrollo social de toda la nación. Cuando una sociedad carece de un espíritu crítico que le permita rebelarse contra la falta de políticas y estructuras informacionales, sólo resta, para cambiar la situación existente, el apoyo decisivo de los profesionales de la información.

El trabajo con comunidades marginadas exige mucho esfuerzo, comprensión, liderazgo y el uso de metodologías adecuadas. El empresario, el académico, el ciudadano incluido en la sociedad de la información, deben encarar ese tipo de trabajo como un desafío, y, ante todo, como un rescate de su deuda social. La falta de lectores, de ciudadanos críticos, se debe según los sociólogos al hecho de que hayamos pasado rápidamente de una cultura oral a una cultura audiovisual, fenómeno que enfatiza la necesidad del mediador en el proceso de inclusión.

En el Brasil, y no obstante los enormes avances económicos ocurridos, se dejaron de realizar reformas y de tomar decisiones políticas que impidieran el enfrentamiento de los problemas asociados a la concentración de riqueza y a la exclusión social. Como consecuencia, la manipulación de conceptos tan simplistas como el de estar o no alimentado, ser o no alfabetizado, tener o no una ocupación con rendimiento adecuado, comenzó a mostrarse insuficiente para abordar el proceso de exclusión. También se tornó inadecuada la simple enumeración cuantitativa del acceso a la educación, al trabajo, a la información, etc., debiendo acoplar la noción de calidad del acceso a los bienes y servicios públicos.

El concepto de exclusión social está siendo adoptado, de manera creciente, para dar cuenta de un fenómeno que se manifiesta en forma cada vez más compleja.

En el panorama que se anuncia, no puede aceptarse ya la exclusión ni la simple aceptación de la miseria. Integrar a los excluidos es la semántica de la solidaridad. No obstante, las actividades antisolidarias aún encuentran eco y condiciones favorables para su proliferación. Reman contra esa corriente las apuestas a favor del actual desarrollo tecnológico y de las redes interactivas que lo acompañan, multiplicándose en redes de solidaridad.

La dimensión social del conocimiento juega un papel central en ese proceso. Representa la alianza entre la academia, el gobierno, la iniciativa privada y la sociedad civil organizada. EI papel de la academia es fomentar la investigación, crear bases teóricas, desarrollar metodo- logías adecuadas y conocimientos que contribuyan a la solución de los problemas nacionales. El papel del Gobierno es formular políticas públicas, crear una infraestructura para favorecer la inclusión social y la difusión del conocimiento. El papel de la iniciativa privada es el rescate de su responsabilidad social. El papel del Tercer Sector es facilitar las acciones de todos los actores antes citados. Es ésta la única alianza que puede generar un esfuerzo común capaz de implantar una política de justicia social, de crear una conciencia solidaria abocada a minimizar las desigualdades sociales, y de ampliar en el Brasil la sociedad de la información.

\section{Referencias}

Castells, Manuel. Flujos, redes e identidades: uma teoria crítica de la sociedad informacional. // Actas del congreso internacional. Nuevas perspectivas críticas en educacion. Barcelona: Paidós, 1994, p.25.

Castells, Manuel. The power of identity - the information age: economy, society and culture. Oxford: Blackwell, vol. 2, 1997, p.103.

Eigen, Manfred. O que restará da biologia do século $X X$ ? // Michel P. Murphy \& Lucke J.O'Neil, org. O que é vida? 50 anos depois. São Paulo: Unesp, 1997, p.33.

Lévy, Pierre; Authier, Michel. A inteligência coletiva; por uma antropologia do ciberespaço. São Paulo: Loyola, 1998, p.65.

Oliveira, Cecília Leite. Biblioteca pública centro de convergente das aspirações comunitárias: Serviço de informação à comunidade nas bibliotecas públicas do Distrito Federal. Brasília: Universidade de Brasília, 1996, p.77 (Dissertação de mestrado).

Oliveira, Cecília Leite. A revolução tecnológica e a dimensão humana da informação: a construção de um modelo de mediação. Brasília: Universidade de Brasília, 2002. (Tese de doutorado).

Suaiden, Emir. El impacto social de la lectura. De antologia. Asociación Colombiana de Lectura y escritura, Bogotá Colômbia, 2003. 2:81-90.

Suaiden, Emir. The social impact of public libraries. // Library Review. London. 52, n 8 (2003) 379-387.

Suaiden, Emir. A biblioteca pública no contexto da sociedade da informação. // Ciência da informação. Brasília. $20: 2$ (2000) 52-60.

Tarapanoff, Kira; Suaiden, Emir; Oliveira, Cecilia Leite. Funções Sociais e oportunidades para profissionais da informação. // Datagramazero Revista (eletrônica) de Ciência da Informação. Rio de Janeiro. 3:5 (2002). 